\title{
Case Report of a Superior Mesenteric Artery Syndrome in an Autistic Patient with Schizophrenia
}

\author{
Lee Mem Tim*, Bernard Ho Kar Eng, Sentilnathan Subramaniam, Harivinthan Sellappan
}

\section{Department of General Surgery, \\ Queen Elizabeth Hospital I, \\ Kota Kinabalu, Sabah, Malaysia \\ *Corresponding author's email: memtim2390@gmail.com}

Keywords: mesenteric, schizophrenia, gastric, duodenum

NMRR -20-290-53310
Introduction: Superior mesenteric artery (SMA) syndrome is a rare cause of upper gastrointestinal obstruction. Diagnosis is confirmed via computed tomography (CT) scan showing acute angulation at the origin of superior mesenteric artery compressing on the duodenum causing proximal dilatation of the second part of duodenum. Case Presentation: We reported a 20-year-old male with underlying autism who was recently diagnosed with schizophrenia and started on antipsychotic medication. He presented with sudden onset of epigastric discomfort and recurrent bilious vomiting with sudden weight loss of $8 \mathrm{~kg}$ in 6 months. He was clinically malnourished with a body mass index of 17 but other physical examinations were unremarkable. CT abdomen on admission confirmed compression of the third part of duodenum by SMA resulting in proximal dilatation of oesophagus, stomach and duodenum. The aortomesenteric angle and distance measured $11^{\circ}$ and $4 \mathrm{~mm}$ respectively. An endoscopic-guided naso-jejunal tube was inserted for nutritional build-up followed by a laparoscopic duodenojejunostomy 2 weeks later. Discussion: SMA syndrome represents a diagnostic and therapeutic challenge. Its prevalence is $0.1-0.3 \%$. SMA syndrome can present with an acute occurrence of abdominal distension or discomfort with vomiting. Contrast-enhanced CT diagnostic criteria for SMA syndrome include aortomesenteric angle and distance of less than $22^{\circ}$ and less than $8-10 \mathrm{~mm}$ respectively. Various options 
Borneo Journal of Medical Sciences (BJMS), Special Issue, Volume 4, March 2021: 21 - 22

are described in managing SMA syndrome and literature has reviewed that laparoscopic duodenojejunostomy is a safe and effective operation. Conclusion: We emphasize the importance of considering SMA syndrome as a differential diagnosis in upper gastrointestinal obstruction with bilious vomiting and a history of significant weight loss. 